\title{
Correction to: Multicentric Castleman's disease in HIV patients: a single-center cohort diagnosed from 2008 to 2018
}

\author{
Smaranda Gliga ${ }^{1} \cdot$ Hans Martin Orth ${ }^{1} \cdot$ Nadine Lübke $^{2} \cdot$ Jörg Timm $^{2} \cdot$ Tom Luedde $^{1} \cdot$ Björn-Erik Ole Jensen ${ }^{1}$
}

Published online: 10 June 2021

○) Springer-Verlag GmbH Germany, part of Springer Nature 2021

Correction to: Infection

https://doi.org/10.1007/s15010-021-01618-5

The original version of this article unfortunately contained a mistake. The affiliations were incorrect. The correct information is given below.

Smaranda Gliga ${ }^{1}$, Hans Martin Orth ${ }^{1}$, Nadine Lübke $^{2}$, Jörg Timm $^{2}$, Tom Luedde ${ }^{1}$, Björn-Erik Ole Jensen ${ }^{1}$

${ }^{1}$ Department of Gastroenterology, Hepatology and Infectious Diseases, University Hospital Düsseldorf, Heinrich Heine University, Düsseldorf, Germany.

The original article can be found online at https://doi.org/10.1007/ s15010-021-01618-5.

Smaranda Gliga

smaranda.gliga@med.uni-duesseldorf.de

1 Department of Gastroenterology, Hepatology and Infectious Diseases, University Hospital Düsseldorf, Heinrich Heine University, Düsseldorf, Germany

2 Institute of Virology, Heinrich Heine-University, University Hospital Düsseldorf, Düsseldorf, Germany
${ }^{2}$ Institute of Virology, Heinrich-Heine-University, University Hospital Düsseldorf, Düsseldorf, Germany.

The original article has been corrected. 